Mots. Les langages du politique

\title{
« Il n'y a pas de droite ici au Venezuela »
}

Les interprétations conflictuelles ordinaires des mots du politique

"There is no right, here, in Venezuela". Ordinary conflicting interpretations of political terms

"No existe aquí derecha en Venezuela". Interpretaciones conflictivas ordinarias de las palabras políticas

\section{Clémentine Berjaud}

\section{OpenEdition Journals}

Édition électronique

URL : https://journals.openedition.org/mots/22091

DOI : $10.4000 /$ mots. 22091

ISSN : 1960-6001

Éditeur

ENS Éditions

Édition imprimée

Date de publication : 30 novembre 2015

Pagination : $37-52$

ISBN : 978-2-84788-776-1

ISSN : 0243-6450

\section{Référence électronique}

Clémentine Berjaud, « « II n'y a pas de droite ici au Venezuela » », Mots. Les langages du politique [En ligne], 109 | 2015, mis en ligne le 30 novembre 2017, consulté le 24 avril 2022. URL : http:// journals.openedition.org/mots/22091; DOI : https://doi.org/10.4000/mots.22091 


\section{« Il n'y a pas de droite ici au Venezuela ». Les interprétations conflictuelles ordinaires des mots du politique}

En prenant pour cadre le Venezuela d'Hugo Chávez, cet article s'ancre dans un contexte discursif et politique spécifique. En effet, entre 1998, date de l'arrivée au pouvoir d'Hugo Chávez, et fin 2012, ce pays connaît non seulement de profondes transformations économiques, sociales et politiques (Compagnon et al., 2009; Folz et al., 2013) mais encore trois processus conjoints. Premièrement, la polarisation politique progressive cristallise un certain nombre d'antagonismes, politiques mais aussi liés aux appartenances sociales en conflit dans le pays. L'inclusion dans le champ politique de franges de la population qui en étaient auparavant distantes (Berjaud, Tarragoni, 2012) si ce n'est exclues $^{1}$, devient, ensuite, un phénomène important qui marque par son intensité et sa durée les mandats successifs d'Hugo Chávez. Concernant le contexte discursif enfin, l'usage et la multiplication des prises de parole présidentielles constituent un facteur clef pour comprendre les spécificités du terrain vénézuélien : les discours présidentiels sur la période représentent selon les estimations plus de 3500 heures de transmission télévisée en direct, ce qui fait près de 146 jours de diffusion en heures cumulées pour plus de 2600 discours, soit une moyenne de plus de 185 discours par an (un discours tous les deux jours).

Ces particularités font donc du Venezuela un terrain propice à un déplacement du regard, des discours politiques (nombreux, longs et répétés) prononcés au sommet de l'État à leurs interprétations et usages par les citoyens ordinaires, qui les reçoivent, en discutent, leur donnent un sens et les font circuler dans leurs vies quotidiennes. En prenant le parti d'étudier ce que les récepteurs citoyens font de tels discours, et de ceux d'Hugo Chávez en l'espèce, il semble possible d'apporter un nouvel éclairage sur la construction des

1. Nombre d'habitants de quartiers populaires ou de zones rurales difficiles d'accès ne possédaient pas de papiers d'identité lors de l'arrivée au pouvoir d'Hugo Chávez, et donc ne pouvaient pas voter. Les mises à jour successives des registres électoraux tout comme certaines politiques publiques spécifiques (comme la Mission Identité ou d'autres Missions éducatives et sociales) ont contribué à faire évoluer cette situation pendant cette période.

CESSP-CRPS (Université Paris 1 Panthéon-Sorbonne)

GEIVEN (Groupe d’Études Interdisciplinaire sur le Venezuela)

clementine.berjaud@wanadoo.fr 
identités politiques et sociales (Avanza, Laferté, 2005) par l'étude des appropriations différenciées des mots du politique. Comment les interprétations et les usages de ces mots sont-ils à la fois révélateurs et constitutifs de lignes de fractures et de conflits sociopolitiques dans le Venezuela d'Hugo Chávez?

Pour comprendre comment les mots et les constructions de sens qui leur sont liées sont traversés par les identités et les appartenances de ceux qui s'en saisissent, nous allons en étudier dans un premier temps les appropriations concurrentielles et conflictuelles. Elles constituent des marqueurs et des repères qui servent avant tout à s'auto-désigner (individuellement et collectivement: «nous ») ainsi qu'à désigner l'autre ou les autres («eux»), ce qui permettra, dans un deuxième temps, de les analyser comme ressorts et ressources des affrontements sociopolitiques au Venezuela pendant cette période.

Trois mots (socialisme, gauche et droite) ont été sélectionnés parce qu'ils cristallisent ces affrontements. Très fréquents et largement polémiques dans l'ensemble de notre corpus, marqueurs forts du champ politique vénézuélien et courants dans les discours d'Hugo Chávez (Gonzalez Binetti, 2012), ces trois termes, dans leurs contextes discursifs, présentent l'intérêt de donner à voir comment les positions et les oppositions se construisent relationnellement et processuellement. Ces mots ne seront donc pas analysés en tant que tels ou en rapport avec des situations d'énonciation ${ }^{2}$ au sommet de l'État, mais plutôt tels qu'ils sont, circulent et participent de la construction de camps politiques antagonistes dans la bouche des citoyens ordinaires (Le Grignou, 2003). Ce sont leurs interprétations, ce qu'elles recouvrent ou non, les manières de leur donner du sens et leurs emplois conflictuels, à l'origine de processus liés à la fabrique discursive de l'appartenance et de l'altérité, qui seront analysés ici.

Notre étude s'appuie sur une méthodologie originale et approfondie. Nous avons rassemblé 29 entretiens collectifs ${ }^{3}$ complétés par des observations, des questionnaires et des entretiens individuels semi-directifs, notamment en temps de campagne électorale avec les mêmes enquêtés. Les 103 enquêtés, choisis selon le principe de diversification, se composent de personnes issues de différents milieux sociaux ${ }^{4}$, aux catégories socioprofessionnelles et aux niveaux d'études variés. Le positionnement politique a également été pris en compte tout comme leur degré de politisation (Lagroye, 2003), de compétences politiques (Bourdieu, 1979; Gaxie, 1978) et éventuellement d'engagement (Matonti, Poupeau, 2004). Pour chaque entretien collectif, trois extraits

2. Voir par exemple De Souza, 2009a et 2009b; Peñafiel, 2011 ; Ramirez, 2013.

3. Au sens de Duchesne, Haegel, 2008 ; dont la composition est dite «naturelle», c'est-à-dire relevant de personnes proches (familles, amis, collègues) et échangeant régulièrement ensemble hors de l'enquête. Dans ce cadre, cette méthode permet de mieux saisir les processus de construction de sens, le rôle des interactions ordinaires et les processus de circulation interpersonnelle des mots, des catégories et des représentations.

4. Séjours de longue durée dans un village isolé en milieu rural, dans un bidonville et enfin dans les quartiers habités par les catégories moyennes et supérieures de la population de la capitale. 
de discours présidentiels, actualisés ${ }^{5}$ pour chaque session de terrain, ont servi de support à la discussion, choisis en fonction de plusieurs critères tels que le dispositif discursif et scénique (une arène solennelle, une estrade en plein air, avec ou sans représentation du public, etc.), le ton et le mode d'adresse, l'échelle (du local à l'international) et le contenu (thèmes abordés, vocabulaire, propositions politiques, variations des registres notamment).

\section{Les appropriations concurrentielles des mots du politique}

Gauche, droite et socialisme font tout d'abord l'objet d'interprétations divergentes, politiquement et socialement différenciées par les citoyens. Ces termes en effet ne recouvrent pas pour les récepteurs les mêmes réalités historiques, les mêmes partis politiques, ni les mêmes lignes ou postures idéologiques. Les destinataires de ce travail discursif de relativement longue durée, qui vise à la fois à construire ces catégories, à leur donner un sens ainsi qu'à mettre ce sens en conformité (Bourdieu, 2001), s’approprient celui-ci de manière contrastée. Entre résistances (Hall, 1994 et 2007), obliquités (Hoggart, 1991) et bricolages (Joignant, 2007), il s'avère en effet que ces trois mots ne sont pas interprétés de la même manière par les enquêtés. Ils ne sont d'ailleurs pas interprétés de façon seulement politique. Il s'agit de présenter en termes d'identités et d'appartenances ces diverses modalités de construction sémantique, traversées par des (in)définitions concurrentes au regard du champ politique (Dulong, 2010).

\section{Le socialisme : pluralité et enjeux de définitions}

Premier vocable qui cristallise l'affrontement politique de ces dernières années au Venezuela (Gonzalez Binetti, 2012 ; López Maya, 2011), socialisme prend différentes significations dans la bouche des citoyens. D'une part, ceux-ci l'emploient tous sans exception, ce qui n'est pas le cas de l'expression capitalisme par exemple, largement absente des propos tenus par les enquêtés opposés aux gouvernements successifs d'Hugo Chávez. D'autre part, ce mot se caractérise par une grande diversité de ses emplois et de ses définitions, politiques ou non. Or ces usages sont au cœur de processus de reconnaissance, d'appartenance et de construction de groupes sociaux et politiques.

Ce terme fait tout d'abord l'objet d'interprétations politiques concurrentes, témoignant de l'appropriation par les citoyens des enjeux propres au champ politique institué. Ainsi, dans le cas de personnes fermement opposées aux

5. Extraits des Aló Presidente $n^{0} 309$ ( 27 avril 2008), $n^{\circ} 346$ (20 décembre 2009) et $n^{\circ} 370$ ( 27 février 2011), de discours nationaux (3 décembre 2006,15 février 2009 et 27 février 2011) et de discours sur des thèmes plus internationaux (10 novembre 2007, 16 décembre 2009 et 15 mars 2011). 
politiques menées dans le pays par Hugo Chávez, issues des catégories supérieures de la population, cumulant très fort capital économique et fort capital culturel (Anabella, 18 ans, est étudiante dans une université catholique de renom, Patricia, 38 ans, est avocate), la bataille des mots se donne à voir autour de ce terme :

- Patricia : Alors il [H. Chávez] annonce que le socialisme est arrivé, et qu'il va rester... enfin, il dit le socialisme, mais le mot est communisme, c'est cela qui va rester;

- Anabella : Exact, le socialisme c'est que tous disposent des mêmes chances, une égalité des chances, mais pas une égalité de statut; nous ne pouvons être tous égaux puisque nous ne sommes pas tous les mêmes, nous ne pensons pas pareil, nous n'avons pas les mêmes...

- Patricia :... niveaux de formation...

- Anabella : Donc tu ne peux pas dire «tous égaux» alors que nous ne le sommes pas.

- Patricia : Exact.

- Anabella : Donc, pour moi le socialisme c'est l'égalité des chances. [...] Rien à voir avec ce qu'il dit là. ${ }^{6}$

Ces propos témoignent d’interprétations politiquement situées du terme, par le biais de l'opposition entre égalité des chances et égalité de statut. Pour ces deux femmes, cette différenciation politique et idéologique repose sur la mise en tension d'identités («nous ne sommes pas tous les mêmes», le ton monte et le visage de Patricia exprime le mépris) elle-même largement articulée dans leurs paroles à un processus de distinction sociale liée au niveau d'étude.

À l'autre extrémité du spectre politique et social, Vera (30 ans, commerçante informelle, membre du Parti socialiste uni du Venezuela - PSUV - et d'un comité de santé), Yanisa (33 ans, gardienne, membre du PSUV et d'un comité de santé), Eliana (56 ans, femme au foyer, membre du PSUV et d'un comité de santé, niveau scolaire équivalent au collège) et Becca (47 ans, femme au foyer, membre du PSUV et de la mission «Mère du Barrio », niveau scolaire équivalent au lycée), habitantes d'un bidonville et amies, définissent ainsi ce même terme :

- Vera : Le socialisme!

- Yanisa : Le socialisme c'est l'inclusion...

- Eliana: C'est nous tous!

6. "-Patricia: Entonces, anuncia que el socialismo llegó, y que va a quedarse... pue' dice socialismo, pero la palabra es comunismo, eso es lo que va a quedarse / - Anabella: Exacto, el socialismo es que todos tengan las mismas oportunidades, igualdad de oportunidades pero no igualdad de estatuto; no todos podemos ser iguales pues que no somos todos los mismos, no pensamos igual, no tenemos las mismas... / - Patricia: ... niveles de formación... / - Anabella: Entonces no puedes decir «todos iguales» mientras que no lo somos / - Patricia: Exacto. I - Anabella: Entonces, para mí el socialismo es igualdad de oportunidad [...] nada que ver con lo que dice allí.» 
- Vera : ... alors que le capitalisme, c'est un, et un et l'autre...

- Eliana :... le socialisme c'est tous ensemble et non chacun séparé

- Becca:... que tous parlent, contribuent...

- Yanisa :... soient pris en compte, et la liberté d'expression...

- Becca :... que tous puissent, aient le droit de...

- Yanisa : ... à la démocratie...

- Becca :.... des droits tout courts!

- Vera: Alors que le capitalisme, comme avant, c'est l'exclusion...

- Becca:... l'exclusivité, les intérêts privés...7

Toutes ces femmes sont situées dans les fractions les moins dotées, sur le plan économique, des catégories populaires (sans revenu autre que les aides publiques pour Eliana et Becca, avec des revenus généralement instables et inférieurs au salaire minimum pour les autres); mais elles se distinguent par leurs engagements dans le quartier, et même hors du quartier pour Yanisa (membre d'un syndicat lié à son emploi de gardienne). Leur interprétation à plusieurs voix repose sur différents éléments politiques comme dans le premier cas. On retrouve en effet la mobilisation de mots issus de l'univers politique institué («la liberté d'expression», «la démocratie», «les intérêts privés», etc.) et cette interprétation (plurielle et à plusieurs voix) se construit aussi de manière oppositionnelle, par rapport au «capitalisme» cette fois. L'opposition entre inclusion et exclusion, en revanche, est plus ambiguë : elle peut être considérée comme une utilisation de catégories politiques pour donner du sens au socialisme, mais elle comporte en même temps un deuxième indice, celui de l'importance du sentiment d'appartenance à une communauté sociale et politique.

Dans ces deux exemples, l'emploi à plusieurs reprises du nous («nous ne pouvons être égaux» dans le premier et "c'est nous tous» dans le second) constitue donc un marqueur des luttes sous-jacentes aux énoncés; une forme d'identité politique collective transparaît dans ces constructions oppositionnelles, renforcées par la désignation explicite de l'autre (Moirand, 2012), ceux qui n'ont pas le «même niveau » d'un côté et « les capitalistes » de l'autre. L'appartenance sociale est une clef pour comprendre l'ensemble des interprétations non politiques du mot socialisme, relevées en grand nombre et sous une large gamme de formes dans le corpus.

7. «-Vera: El socialismo! / - Yanisa: El socialismo es inclusión... / - Eliana: Es nosotros todos! / - Vera: ... mientras que el capitalismo es uno, uno y otro... / - Eliana: ... el socialismo es todos juntos, y no cada uno separado / - Becca: ... que todos hablen, contribuyan... / - Yanisa: ... sean tomados en cuenta y la libertad de expresión... / - Becca:... que todos puedan, tengan el derecho de... / - Yanisa: ... a la democracia... / - Becca: ... derechos y ya! / - Vera: Mientras que el capitalismo es como antes, es exclusión... / - Becca: ... la exclusividad, los intereses privados... » 


\section{Le socialisme au-delà des lectures politiques}

\section{Le règne de Dieu sur terre}

- Juanella : C'est clair parce que, pour les catholiques, grâce à Dieu, Jésus-Christ est arrivé, et il a partagé, la nourriture par exemple et tout le reste, donc il était socialiste, c'est le premier socialiste...

- Juana : et même plus... Regarde la bible. Et cet homme-là, celui qui marche à travers les nations, celui qui est là sur cette terre, celui-là c'est Hugo Rafael Chávez Frías .

- Luis : et même plus, je te raconte, je me rappelle, quand ma mère, malade, allait à l'Église pour prier et demander au Christ de guérir... elle ne voyait plus... et les aveugles, les sourds, etc., tous étaient à l’Église, et là, Chávez dit : «Que t’arrivet-il? Crois-tu que nous devions attendre un miracle? Le miracle faisons-le!», et il a fait la Mission Miracle ${ }^{8}$ C'est ça le socialisme camarade! C'est ça le règne de Dieu sur terre! aussi simple que ça! 9

\section{La pêche}

Le socialisme et tout ça, c'est comme la pêche : pour le poisson il n'y a pas de prix pour la communauté [le village], on partage, c'est comme... gratuit. Ensuite il y a un prix pour la coopérative de C. et encore un autre prix pour exporter aux « grands ». $(\text { Pablo })^{10}$

\section{Le prix d'un Hummer}

Le socialisme c'est partager les choses... genre... tu crois qu'on va partager nos voitures, nos maisons? Et les ministres du socialisme? Ils ont de belles maisons et des hummers, tu crois qu'ils partagent? II n'y a pas de socialisme ici, et pas de communisme comme ils disent aussi... (Antonio) ${ }^{11}$

8. La Mission Miracle est une politique publique qui permet aux personnes sans ou à faibles revenus de se faire opérer, notamment de la cataracte, comme ce fut le cas de la mère de Luis.

9. "-Juanella: Claro porque, para los católicos, gracias a Dios, Jesús Cristo llegó, y compartió, la comida por ejemplo, y todo, entonces era socialista, es el primer' socialista... / - Juana: y aún más... mira la Biblia. Y este hombre, el que camina por las naciones, el que está aquí en esta tierra... este es Hugo Rafael Chávez Frías / - Luis: y aún más, te cuento, me acuerdo, cuando mi madre, enferma, iba en la iglesia para rezar y pedir al Cristo de curar... ella no veía... y los ciegos, los sordos etc. todos estaban en la iglesia, y en este momento Chávez dijò: "Que te pasa? Crees que tengamos que esperar un milagro? El milagro hagámoslo!" e hizo la Misión Milagro! esto es socialismo camarada! eso es el reino de Dios en la tierra! tan sencillo como esto! » Entretien réalisé avec une famille de milieu populaire habitant dans un bidonville. La mère, Juana (41 ans), est coiffeuse, le père, Luis ( 43 ans), est artisan et la fille, Juanella (20 ans), poursuit des études à l'Université bolivarienne, créée sous le gouvernement d'Hugo Chávez, faisant la fierté de toute la famille (et des voisins). Ils votent pour Hugo Chávez.

10. "El socialismo y todo eso, es como en la pesca: para el pescado, no hay precio para la comunidad, compartimos, es como... gratuito. Después hay un precio para la cooperativa de C. [nombre del pueblo vecino], y otro otro precio para exportar a los "grandes". » Pablo, 29 ans (pêcheur dans un petit village de la côte nord du pays ; il vote pour Hugo Chávez).

11. "El socialismo es compartir las cosas... tipo... ¿crees que vamos a compartir nuestros carros, nuestras casas?? ¿Y los ministros del socialismo? Tienen lindas casas y hummers, ¿crees que comparten? No hay socialismo aquí, y no comunismo como dicen también... » Antonio, 30 ans (vendeur ambulant dans un bidonville de Caracas; il ne vote pas). 
Ces différentes interprétations ne sont alors pas politiques au sens légitime (Collovald, Sawicki, 1991). Elles font appel à l'expérience sociale, aux expériences personnelles - y compris sous l'angle d'une vision religieuse et matérielle que les locuteurs vivent au quotidien. Néanmoins, ces propos se structurent aussi par le biais d'oppositions : «nous» et la «communauté» contre les «grands » exportateurs pour Pablo, ou les «ministres» pour Antonio. Dans ce cadre, les emplois et les définitions concurrentes de socialisme sont à comprendre d'après les identités sociales, d'une part, exprimées ici par le sentiment d'appartenance à un même groupe (Charaudeau, 2009b) ou non, appartenances qui se traduisent dans leurs expériences quotidiennes. Elles résultent d'autre part de leurs orientations politiques, de leurs votes et de leurs partis pris en particulier. Dans le cas du Venezuela comme ailleurs ${ }^{12}$, en effet, les positions sociales sont en lien avec les positionnements politiques.

\section{Gauche et droite : de la lecture compétente auxpositionnements inattendus}

Quant aux catégories politiques relatives aux désignants droite et gauche, elles suscitent également plusieurs modalités d'appréhension par les citoyens (Gaxie, 2012). Certains, en premier lieu, développent une définition politique et politiquement située de ces termes. Compétents politiquement (Bourdieu, 1979), comme Mauricio, 35 ans, ingénieur et militant de longue date, aujourd'hui au PSUV, ils mobilisent, différencient et manient facilement les deux vocables :

Ici, dans les discours [d'H. Chávez], on parle de droite et de gauche, et c'est une bonne chose, je veux dire, cela permet de différencier plus clairement les options pour les électeurs, c'est nécessaire parce qu'eux [l'opposition] mentent là-dessus. En vrai, à gauche il y a le Pôle patriotique de Chávez, qui est composé de plusieurs partis et mouvements sociaux, puis de plus en plus à droite, $A D^{13}$, Nuevo Tiempo ${ }^{14}$, qui est issu d'une scission avec AD, Copei ${ }^{15}$, Primero Justicia ${ }^{16}$ et Voluntad Popular ${ }^{17}$, eux c'est l'extrême droite, venus d'une scission avec PJ. ${ }^{18}$

12. Et peut-être plus encore qu'ailleurs (Ellner, 2008).

13. Acción Democrática, parti situé au centre gauche durant la Quatrième République, en cours de repositionnement vers le centre droit dès les années quatre-vingt.

14. Littéralement «Un Nouveau Temps», parti issu d'une scission de l'aile libérale d'AD.

15. Parti lié à la Démocratie chrétienne.

16. «La Justice d'abord» : organisation née d'une scission de l'aile la plus conservatrice de Copei.

17. «Volonté populaire » : ce parti est né de conflits internes à Primero Justicia; il représente un courant positionné à l'extrême droite, notamment si on le compare avec le cas français.

18. "Aquí, en los discursos [de H. Chávez], se habla de derecha y de izquierda, y eso es algo bueno, quiero decir, esto permite diferenciar más claramente las opciones para los electores, es necesario porque ellos [la oposición] mienten en eso. En verdad, a la izquierda hay el polo patriótico de Chávez, que está compuesto con varios partidos y movimientos sociales, después, cada vez 
Mauricio décompose donc l'échiquier politique de manière précise, relevant les scissions successives qui ont donné naissance aux partis les plus récents, membres de la coalition d'opposition. Il les classe tous les cinq «à droite", tout en les situant les uns par rapport aux autres jusqu'à «l'extrême droite». Concernant les partis d'opposition, il considère «qu'ils mentent ». Du point de vue des partisans d'Hugo Chávez bien dotés en compétence politique spécifique ${ }^{19}$, donc, l'opposition équivaut à la droite.

En revanche, du point de vue des opposants à Hugo Chávez, la même expression relève le plus souvent du «centre gauche », comme le montre cette remarque de Maria, administratrice culturelle d'un quartier huppé de Caracas :

Moi, je suis de centre gauche, comme l'opposition, il faut dire qu'il n'y a pas de droite au Venezuela, il n'y a jamais eu de droite comme en Argentine ou au Chili. Ici, c'est des populistes ou des sociaux-démocrates. Et quand ils disent qu'on est à droite, c'est un mensonge, c'est du centre gauche, leur but c'est d'aggraver les divisions entre les Vénézuéliens pour mieux gagner, c’est discréditer l'autre, c'est-àdire nous. ${ }^{20}$

Dans ces propos exempts d'hésitations, Maria, 35 ans, interprète tout autrement ces catégories : pour elle, l'opposition est «du centre gauche », " socialdémocrate» plus précisément, et elle se reconnaît dans ce positionnement («moi »). Au vu de sa trajectoire politique d'engagement progressif en faveur de la coalition d'opposition, elle a acquis un certain nombre de connaissances politiques tout en étant, de par ses propriétés dès le départ fortement dotée en capital culturel. Or, ce sont ces éléments qu'elle investit dans son interprétation politique du mot droite, qui lui évoque l' «Argentine ou [le] Chili» et n'est pas pertinent au Venezuela; et du mot gauche, qui réfère aux « sociauxdémocrates », comme elle. Elle rejette alors, sous l'étiquette populiste, la candidature d'Hugo Chávez hors de cette catégorie et accuse de mensonge, comme avant elle Mauricio, le camp opposé à ses propres opinions.

Dans d'autres cas de figure, en milieu populaire ou pour certaines franges de la population peu dotées en compétences politiques spécifiques (ce qui n'empêche pas d'autres formes de politisations, pratiques notamment), ces termes suscitent des hésitations, des ralliements après discussions ou encore de nombreuses non-réponses. Parmi les personnes interrogées, moins d'un enquêté sur cinq répond à cette question posée par questionnaire ${ }^{21}$. Moins

más a la derecha, $A D$, Nuevo Tiempo, que nació de una cisión con $A D$, Primero Justicia y Voluntad Popular, ellos son la extrema derecha, por una cisión con PJ.»

19. Au sens de Bourdieu, 1979.

20. «Yo, estoy al centro-izquierda, igual como la oposición, hay que decir que no hay derecha en Venezuela, nunca hubo derecha como en Argentina o en Chile. Aquí son populistas o socialdemócratas. Y cuando dicen que somos a la derecha, es mentira, es centro-izquierda, la meta es alargar las divisiones entre los venezolanos para ganar mejor, es descreditar al otro, es decir a nosotros."

21. «Où vous positionneriez-vous sur cet échiquier politique? De gauche à droite». À titre de compa- 
d'un sur dix ne coche aucune case et inscrit «Pour Chávez» ou «Chávez » en réponse à la question. Au-delà d'un petit nombre d'enquêtés qui refusent cette catégorisation (de l'ordre d'un survingt), la plupart de ces non-réponses ou de ces réponses manuscrites témoignent de la difficulté de la question, de la méconnaissance de ces catégories mais aussi de la malléabilité des significations que l'on peut leur associer, elle-même révélatrice d'antagonismes forts. Plus encore, ils semblent se tromper parfois.

En croisant ainsi les données recueillies par questionnaire et les propos tenus en entretiens individuels et collectifs, six cas posent question. Ils s'autopositionnent à droite mais soutiennent Hugo Chávez. Ces deux positions ne sont pas incompatibles bien entendu, c'est d'ailleurs le cas de trois autres personnes qui maintiennent cette double position et pour lesquelles elle paraît cohérente au vu de leurs propos en entretien; mais pour ces six enquêtés en particulier, un désajustement s'opère. Ce point attire l'attention dans la mesure où nous classerions ces personnes à gauche selon l'usage usuel du terme. Après plusieurs sollicitations explicites, en entretien comme lors de discussions plus informelles, et n'ayant toujours pas trouvé de réponse ou d'explication à cet auto-positionnement, une voisine du village nous a finalement apporté la clef probable de ce désajustement. Elle explique en effet au détour d'une conversation qu'elle aussi est à droite, comme « tout le monde ici », et se justifie en expliquant qu'elle votait pour AD avant l'arrivée au pouvoir d'Hugo Chávez, donc «à gauche» selon ce classement hérité de la Quatrième République. Or, AD faisant partie de l'opposition aujourd'hui, elle se positionne contre lui, dans l'autre camp, c'est-à-dire très logiquement pour elle «à droite ».

Cette conversation permet de comprendre autrement le fort taux de nonréponses et plus encore les erreurs d'auto-positionnement : elles ne sont pas le simple reflet d'une incompétence politique spécifique, elles constituent plutôt le résultat de formes de compétence à éclipse ${ }^{22}$, liée à la mémoire collective des mots (Moirand, 2007), tout aussi éclairante pour ces électeurs (AD étant à gauche, je suis contre, donc à droite) au moment d'interpréter et de manier ces catégories de l'univers politique institué. En ce sens, c'est bien l'appartenance à un camp que symbolisent ces termes de droite et de gauche, au-delà de la qualification d'une ligne politique. Ces mots marquent alors bien plus, par leurs emplois variés, la polarisation et l'identification à un camp plutôt qu'un contenu programmatique (Bacot, 2011).

Les usages et la référence des vocables droite et gauche pour les enquêtés sont donc révélateurs de l'affrontement discursif symbolique en cours au Venezuela pendant cette période. Chacun interprète ces termes en fonction de

raison, seuls quelques-uns ne répondent pas à la question du soutien au gouvernement Chávez posée dans le même questionnaire : «Soutenez-vous le processus révolutionnaire en cours au Venezuela?»

22. Au sens de Hoggart, 1991. 
sa position politique et sociale (comme pour les retraductions concrètes du socialisme plus haut). Mais le maniement et la circulation des mots du politique reflètent également des lectures particulières de la réalité sociopolitique du pays, fortement polarisée, où deux camps sont en conflit et au sein de laquelle l'on appartient à l'un ou à l'autre. L'identification prime alors sur le nom ou la référence idéologique qu'il porte (socialisme, droite ou gauche). Ces termes constituent donc des marqueurs ou des repères servant à s'auto-désigner et à désigner l'autre. Ils tracent une frontière et témoignent des conflits qui se jouent entre appartenance et altérité (Silva, 2004) ; les citoyens se les approprient ainsi de manière différenciée et s’identifient ou non aux groupes et aux acteurs politiques qui les représentent.

\section{Ressorts et ressources d'affrontements sociopolitiques}

Le vocabulaire permet alors de comprendre les ressorts des affrontements discursifs et symboliques les plus saillants ces dernières années au Venezuela (Bolivar, 2007), en ce qu'ils apparaissent comme des ressources, voire des armes, dans les conflits sociopolitiques passés et parfois encore actuels.

\section{La construction de l'appartenance : une ressource électorale}

Dans ses nombreux discours, Hugo Chávez associe le socialisme, mis en avant idéologiquement depuis 2005 (López Maya, 2013), et l'appartenance au peuple. En rattachant ce terme à un collectif légitimant qu'il cherche à faire exister (Ricoeur, 1990), l'ancien président vénézuélien construit une autre forme d'appartenance, parallèle et liée à la construction d'un électorat. Du côté des électeurs, cette correspondance fait sens, qu'ils soient favorables ou opposés au gouvernement, comme pour Ariadna, cette étudiante qui souligne le lien entre politique menée et groupe social :

[C'est] comme des «telenovelas», pour le peuple, pour que les gens s'identifient à sa personne [...] sa manière de parler... c'est un show médiatique mais, au final, il ne dit rien. ${ }^{23}$

Ariadna est une étudiante en communication de 21 ans, issue des catégories sociales supérieures ${ }^{24}$ et a participé à plusieurs manifestations de l'op-

23. "Igual como en las telenovelas, para el pueblo, para que la gente se identifique a su persona [...] su manera de hablar... es un show mediático, pero al final, no dice nada. "

24. Sans connaître la profession de ses parents, on peut toutefois se référer ici au quartier qu'elle habite avec ses parents et ses frères et sœurs (cinq enfants en tout), qui constitue l'un des quartiers les plus chers de la capitale. Tout proche du centre économique et du quartier des affaires, cette zone s'organise en larges avenues arborées et rectilignes, chacune d'entre elles desservant tout au plus une quinzaine de villas avec jardins ou parcs, toutes entourées de fils barbe- 
position. Les images de l'extrait ont d'abord attiré son attention, elle les interprète comme "un show médiatique » : la mise en scène familiale et les tenues la font penser aux telenovelas, genre télévisuel populaire qu'elle méprise. Elle note ensuite l'accent et l'intonation d'Hugo Chávez ("sa manière de parler»), très différents des siens, qu'elle renvoie implicitement à l'hexis c'est-à-dire à une appartenance sociale, ici opposée à la sienne. Dans son cas, si elle reconnaît le mécanisme de représentation, elle rejette donc l'appartenance à ce collectif représenté : le peuple, c'est les autres. Elle poursuit quelques minutes plus tard dans l'entretien, refusant ce processus d'identification :

Quand il dit, quand il fait, c'est pour les pauvres, pour ceux qui sont pas allés à l'école, qui n'ont pas d'éducation; pas comme nous, pas pour nous qui avons eu ces opportunités. ${ }^{25}$

L’appartenance sociale se décline entre eux, «ceux qui ne sont pas allés à l'école» et nous, ceux qui "[ont] eu ces opportunités». La négation et la violence symbolique de ses propos sont au cœur de la phrase, c'est par ce biais qu'elle donne du sens aux mots prononcés. Bien qu'elle rejette «le peuple» et les "pauvres» en tant que collectifs socialement constitués auxquels elle ne se sent pas appartenir, ces interprétations, qu'elles soient nées de l'image, du contenu du discours ou de l'orientation politique du dirigeant, procèdent du même type de mécanismes : elle met en relation son identité, en particulier sociale, avec celle qu'elle attribue au locuteur pour comprendre les mots du discours. Le conflit politique va donc de pair avec la mise en tension des identités sociales non seulement que les enquêtés perçoivent, mais encore auxquelles ils s'identifient ou non (Lozada, 2003). Dans ce cadre, construire discursivement des groupes sociaux renvoie du même coup aux électorats. L'identification sociale constitue donc une ressource électorale dans le premier cas, et un ressort de l'exacerbation des affrontements (sociaux) dans le second.

\section{Une arme politique: (dé)qualification et disqualification}

Les luttes discursives portant sur le sens de ces termes, socialisme, gauche et droite, se cristallisent enfin en temps de campagne électorale; il ressort en effet des entretiens effectués à cette période une différenciation interprétative accrue $^{26}$ et plus conflictuelle encore. Du côté des partisans d’Hugo Chávez,

lés et de clôtures électriques hautes de plusieurs mètres. On sait par ailleurs que deux domestiques au moins vivent avec eux.

25. "Cuando dice, cuando hace, es para los pobres, para los que no fueron a la escuela, que no tienen educación; no como nosotros, no para nosotros que tuvimos estas oportunidades. "

26. Les propos ci-dessous sont issus des entretiens individuels réalisés en août 2012 durant la campagne électorale où l'utilisation des termes de gauche et droite augmente substantiellement dans les discours des deux principaux candidats, ce que nos enquêtés ont remarqué (l'usage du mot socialisme reste élevé et stable quant à lui). 
droite sert ainsi en premier lieu à désigner et à disqualifier l'autre, l'opposition $^{27}$ sous toutes ces formes; comme pour Alvaro, étudiant :

À l'heure de choisir et de voter, il faut toujours revenir aux fondamentaux : d'un côté il y a les humanistes, ceux qui sont pour le bien commun, pour la société et le collectif, c'est la gauche et c'est le PSUV. De l'autre, on a l'individualisme, l'exploitation de l'autre et les bénéfices personnels, c'est la droite; et même si eux proposent leur définition propre, genre 'nous sommes le centre', 'nous sommes l'unité' tout ça, il ne faut pas se leurrer, il n'y a pas de centre au Venezuela, tu dois choisir en fonction de ça. ${ }^{28}$

Alvaro, 26 ans, étudiant issu des catégories populaires et favorable à Hugo Chávez, dénonce ici les discours de l'opposition qui, selon lui, cherche à se faire passer pour une coalition centriste. Cette fois-ci, «il n'y a pas de centre au Venezuela». En déconstruisant le discours porté par les principaux acteurs de la coalition sur ce point, il les disqualifie, au sens propre comme au sens figuré.

Dans nombre de cas, ce décryptage qui reproduit la confrontation politique se retrouve. Celle-ci est d'ailleurs parfois directe, comme lors de discussions collectives où l'affrontement des processus de (dé)qualification s'exprime de façon explicite. Cilena et Andrea, deux amies de Maria, bien dotées en capital culturel et économique, discutent ainsi :

Cilena : Moi je suis de centre gauche vois-tu, nous avons des différences idéologiques certes, mais il n'y a pas de droite ici au Venezuela depuis le Pacte de Punto Fijo, donc je suis à gauche, mais plutôt centre gauche...

Andrea : Ah non! Ici il y de la droite qu'est-ce que tu dis!? Et les vieux de la Quatrième [République]? Ceux qui veulent y revenir? Évidemment ils disent qu'ils ne sont pas à droite, mais c'est un déguisement, c'est la manière dont ils veulent apparaître!

Cilena : Non. Ils ne sont pas à droite, aucune droite, c'est la gauche, bien sûr pas celle de Marx, qui est utopique, mais de gauche tout de même !29

Cilena, 42 ans, autrefois administratrice dans un ministère, favorable à Henrique Capriles après une trajectoire politique d'éloignement du PSUV corrélée à

27. Sur le rôle des mots dans l'entretien de cette polarisation conflictuelle exacerbée, voir aussi Erlich, 2005.

28. "A la hora de elegir y de votar, siempre hay que ver de nuevo los fundamentos: por un lado los humanistas, los que están a favor del bien común, por la sociedad y el colectivo, eso es la izquierda y es el PSUV. Por otro lado, tenemos individualismo, la explotación del otro y los beneficios personales, eso es la derecha; y aun cuando proponen su definición propia, tipo "nosotros somos el centro", "nosotros somos unidad" ytal, no hay que equivocarse, no hay centro en Venezuela, tienes que elegir con respecto a eso. »

29. "Cilena: Yo soy de centro-izquierda ves, tenemos diferencias ideológicas cierto, pero no hay derecha aquí en Venezuela desde el Pacto de Punto Fijo, entonces, soy de izquierda, pero más centro-izquierda... / Andrea: iAy no ¡Aquísi hay derecha ¿qué dices?! ¿y los viejos de la Cuarta? ¿Los que quieren regresarse? ;Obvio que dicen que no son de derecha, pero es como disfrazados, es la manera con la cual quieren aparecer! / Cilena: No. No son de derecha, ninguna derecha, es izquierda, por supuesto no la de Marx, que es utópica, ipero izquierda también!» 
sa trajectoire professionnelle, et Andrea, 37 ans, en cours d'éloignement politique mais encore rattachée au secteur public par sa profession (architecte au sein de la Mission Logement), s'affrontent ici sur le sens à donner à ces termes. Pour la première, comme pour Maria plus haut, «il n'y a pas de droite au Venezuela », l'opposition est, comme elle, de "centre gauche». À l'appui de cette opinion, elle évoque un événement historique, le Pacte de Punto Fijo, acte de naissance de la Quatrième République après des années de dictature militaire. Elle précise sa pensée en évacuant la définition «marxiste» de la gauche, mais s'affirme en haussant la voix «de gauche tout de même».

Face à elle, Andrea répond sur le terrain de la Quatrième République dont les «vieux» dirigeants, qui veulent «revenir» au pouvoir, sont bel et bien de droite pour elle. Elle mobilise à son tour l'idée de manipulation ou de dissimulation par le biais du terme déguisement. La proximité des interprétations des uns et des autres au regard de leur positionnement politique est alors frappante, et ces manières de donner du sens - ou plus précisément de s'affronter sur le sens de ces catégories - confirment l'ambivalence de ces mots du politique (Michelat, 1990). Termes à interpréter, ils constituent aussi des ressources interprétatives et, au-delà, des ressources politiques dans l'affrontement qui se poursuit au Venezuela. La bataille discursive au plus haut sommet de l'État trouve donc une traduction concrète dans les interprétations des citoyens confrontés à ces discours; toutefois, la prégnance du vocabulaire spécifiquement mobilisé par Hugo Chávez, sur «la gauche » ici comme sur «le socialisme» plus tôt, semble restreindre l'espace de cette lutte. Elle se joue à gauche, aucun enquêté partisan d'Henrique Capriles ne se réclamant explicitement de la droite lors de cette campagne électorale, tout comme ils ne se posaient pas en défenseurs du «capitalisme» lors des entretiens collectifs. Entre armes, ressorts et ressources polémiques, ces trois mots recouvrent donc des identités sociales et politiques en confrontation dans le Venezuela d’Hugo Chávez.

En partant de l'usage de trois termes particuliers, socialisme, gauche et droite, cet article a permis de mettre en lumière le lien entre maniement discursif des catégories partisanes, d'une part, et affrontements sociopolitiques de l'autre. En démontrant tout d'abord que ces désignants suscitaient des interprétations divergentes, politiquement et socialement, l'analyse a exploré leur(s) sens du point de vue des citoyens ordinaires. Entre appropriations proprement politiques et autres formes de coconstructions de sens, il apparaît que ces mots sont pris, retraduits, et appréhendés de diverses manières par les enquêtés. Mais ces appropriations reposent toujours sur une appartenance, elles passent par le nous. Au-delà de leurs divergences en effet, on s'aperçoit que les interprétations reliées à ces termes sont concurrentielles dans l'espace des possibles discursifs et interprétatifs. Quel que soit le nom qu'on leur donne, gauche ou droite par exemple, vocabulaire qui n'est d'ailleurs pas 
maitrisé de façon équivalente par ces citoyens, c'est l'identification à un camp qui compte et qui fait sens dans leurs propos.

Dans ce cadre, ces mots servent avant tout à s'auto-désigner et à désigner l'autre. Au fondement cette construction d'appartenances antagonistes, les identités sociopolitiques véhiculées par les discours se retrouvent chez les citoyens et, plus encore, participent de la formation d'électorats. En cela, la mise en tension de ces identités constitue une ressource politique et un ressort primordial des affrontements étudiés. Ces mots sont vecteurs de conflits, en même temps qu'ils en sont les armes symboliques, comme le démontrent en particulier les (dé)qualifications et les disqualifications repérées et leurs usages par les citoyens ordinaires.

En renversant le regard, de l'analyse des discours du pouvoir à l'étude de leurs appropriations effectives par les destinataires, ces éléments théoriques et méthodologiques permettent de comprendre comment les mots et les constructions de sens qui leurs sont liées sous-tendent, révèlent et contribuent aux identités et aux appartenances conflictuelles dans le débat public.

\section{Références}

Avanza Martina, LAfERTE Gilles, 2005, «Dépasser la construction des identités ? Identification, image sociale, appartenance», Genèses, n 61 (4), p.134-152.

BACOT Paul, 2011, La construction verbale du politique, Paris, L'Harmattan.

BERJAUd Clémentine, TARRAGONI Federico, 2012, «Un peuple peut-il survivre à son président? Représentations de l'élection en milieu populaire », Problèmes d'Amérique Latine, ${ }^{\circ} 86$, p. 61-74.

Bolivar Adriana, 2007, «El análisis interaccional del discurso. Del texto a la dinámica social », Análisis del discurso. Por qué y para qué, A. Bolívar éd., Caracas, Los libros de El Nacional.

Bourdieu Pierre, 1979, La distinction. Critique sociale du jugement, Paris, Minuit.

- 2001, Langage et pouvoir symbolique, Paris, Le Seuil.

Charaudeau Patrick, 2005, Le discours politique. Les masques du pouvoir, Paris, Vuibert.

- éd., 2009a, Identités sociales et discursives du sujet parlant, Paris, L’Harmattan.

- 2009b, «Identité linguistique, identité culturelle. Une relation paradoxale», Le discours sur les «langues d'Espagne». El discurso sobre las «lenguas españolas» 1978-2008, C. Lagarde éd., Perpignan, Presses universitaires de Perpignan.

Collovald Annie, SAWICKI Frédéric, 1991, "Le populaire et le politique. Quelques pistes de recherche en guise d'introduction », Politix, $\mathrm{n}^{\circ}$ 13, p. 7-20.

Compagnon Olivier, Rebotier Julien, Revet Sandrine éd., 2009, Le Venezuela au-delà du mythe. Chávez, la démocratie, le changement social, Paris, L'Atelier.

DE SouzA Serge, 2009a, Peuple et populisme dans l'Amérique latine du «tournant à gauche ». Hugo Chávez et Evo Morales (1999-2010), en ligne : http://adispal.edispal.com/2009/10/peuple-chavez-evo-morales.html. 
- 2009b, Bolivar et le bolivarisme dans le discours d'Hugo Chávez (1999-2006), en ligne : http://adispal.edispal.com/2009/10/bolivar-chavez.html.

DucheSne Sophie, Haegel Florence, 2008, L'entretien collectif, Paris, Armand Colin.

ElLner Steve, 2008, Rethinking Venezuelan Politics. Class, Conflict, and the Chávez Phenomenon, Boulder, Lynne Rienner Publications.

ERLICH Francés, 2005, «La relación interpersonal con la audiencia. El caso del discurso del presidente venezolano Hugo Chávez», Signos, n³ 38 (59), p. 287-302.

Folz Olivier, Fourtane Nicole, Guiraud Michèle éd., 2013, Le Venezuela d'Hugo Chávez. Bilan de quatorze ans de pouvoir, Nancy, Presses universitaires de Nancy.

GaXie Daniel, 1978, Le cens caché. Inégalités culturelles et ségrégation politique, Paris, Le Seuil.

- 2012, "Droite ou gauche? Usages et non-usages d'instruments courants d'orientation politique», Gauche/Droite. Genèse d'un clivage politique, J. Le Bohec, C. Le Digol éd., Paris, Presses universitaires de Franc.

Gonzalez Binetti María Fernanda, 2012, Hugo Chávez et Alvaro Uribe ou la force des mots. Deux discours pour gouverner, Paris, L'Harmattan.

HalLStuart, 1994 (1977), «Codages-décodages», Réseaux, nº 68, p. 27-39.

- 2007, Identités et cultures, politiques des Cultural Studies, Paris, Éditions Amsterdam.

Hog GART Richard, 1991, La culture du pauvre, Paris, Minuit.

JoIgNANT Alfredo, 2007, "Compétence politique et bricolage. Les formes profanes du rapport au politique », Revue française de science politique, vol. 57, $\mathrm{n}^{\circ}$ 6, p. 799-817.

LAGROYE Jacques éd., 2003, La politisation, Paris, Belin.

LE Grignou Brigitte, 2003, Du côté du public, Usages et réceptions de la télévision, Paris, Economica.

LóPEZ MAYA Margarita, 2011, «Hacia el socialismo del siglo XXI : los consejos comunales, sus cambios conceptuales y las percepciones de los participantes en Caracas», Ciudadanía y legitimidad democrática en América Latina, I. Cheresky, F. Mayorga, S. Gómez Tagle éd., Prometeo, Buenos Aires, p. 221-262.

- 2013, "La expresión Poder Popular en la era de Chávez (1999-2011)», Séance de clôture du séminaire GEIVEN, «Le populaire en perspective, une approche multidisciplinaire », 10 juin 2013, Paris.

LozAdA Mireya, 2003, "El otro es el enemigo. Imaginarios sociales y polarización», Revista Venezolana de Economía y Ciencias Sociales, vol. IX, n² 2, p.195-210.

MATONTI Frédérique, Poupeau Franck, 2004, "Le capital militant. Essai de définition », Actes de la recherche en sciences sociales, $\mathrm{n}^{\circ} 155$ (5), p. 4-12.

MichelAT Guy, 1990, "À la recherche de la gauche et de la droite», L'électeur français en question, D. Boy, N. Mayer éd., Paris, Presses de Sciences Po, p. 71-105.

MoIRAND Sophie, 2007, Les Discours de la presse quotidienne. Observer, analyser, comprendre, Paris, Presses universitaires de France.

- 2012, "Préface », La médiatisation de la crise du Chikungunya dans l'Océan Indien. Autour des concepts d'altérité, d'interdiscursivité et de responsabilité, Fernelmont, EME, p.11-21.

Penafiel Ricardo, 2011, «L'image du peuple. Construction de l'ethos plébéien de Hugo 
Chávez dans l'émission Aló Presidente», Mots. Les langages du politique, n 96 , juillet, p. 29-44.

RAMIREZ Lilia, 2013, «Representación e identidades colectivas en el discurso político y mediático venezolano : caso Aló Presidente »,Communication présentée lors de la Journée d'étude 2013 de l'Association ADAL (Analyse des discours de l'Amérique Latine) : Identités Sociopolitiques en conflit, 13 juin 2013, Paris.

Ricoeur Paul, 1990, Soi-même comme un autre, Paris, Le Seuil.

SILVA Carlos, 2004, "Dos veces otro. Polarización política y alteridad », Revista Venezolana de Economía y Ciencias Sociales, vol. X, n², p.129-136. 